societies, this is the first major single-author monograph on the topic for more than a decade. We found little to criticize, even in such a large book. Perhaps graphical representations of data might have improved the strength of certain scientific arguments, in place of some of the line drawings, which seem oddly placed and in many cases have no clear connection to the text. On the plus side, many of the taxa covered in the book are illustrated with stunning colour photographs.

Costa takes a refreshingly unbiased approach to examining these fascinating societies, providing insight into how non-eusocial insect societies are structured, as well as details of their systematics, natural history, ecology and anatomy. His writing is scholarly enough to appeal to professionals, yet it is accessible enough to enlighten any interested reader. His book gives the 'other insect societies' welldeserved time in the spotlight, and will be a valuable and appealing addition to the libraries of academics and readers of scientific natural history alike.

Jenai Milliser and George Uetz are in the

Department of Biological Sciences,

University of Cincinnati, Cincinnati,

Ohio 45221, USA.

\section{Beyond fractals}

\author{
Superfractals: Patterns of Nature \\ by Michael F. Barnsley \\ Cambridge University Press: 2006. \\ 464 pp. $£ 25, \$ 35$
}

\section{J. R. Mureika}

Since Benoit Mandelbrot first brought fractals to the attention of the world, their self-similar mystique has wormed its way into the physical, biological and actuarial sciences, as well as psychology and abstract art. Describing the seemingly disordered world with such geometrical simplicity has long been a target of reductionism. However, using simple fractals hasn't been entirely successful: the world isn't quite that easy to describe with algebra.

Anyone whose research involves chaos and recursion will already be familiar with Michael Barnsley's seminal work Fractals Everywhere

(Academic Press, 1988). A collaborative effort with researchers at the Australian National University has led to the development of superfractals, allowing Barnsley to offer a new link between the physical realities of the Universe and the symmetries of mathematics.

Briefly, a superfractal is the result of an iterative application of randomly selected but welldefined transformations to a picture - a hybrid recursion that results in a wide range of beautifully intricate structures. Superfractals colourfully conveys to the reader this next step in chaotic harmony, highlighting the unification of information theory and physics. New to the fractal arena is the use of code space mappings, fractal tops and colour stealing, in which the pixels of the resulting fractallike attractor are assigned hues 'stolen' from a base image.

The book is not easy for lay readers, although the author states that it should be accessible to anyone with a basic understanding of under-

graduate mathematics. The overall structure of the text is well thought out, and at the outset of each chapter the author delineates exactly which salient issues will be addressed in the following pages, making for a good executive summary. But because the book is very linear in its presentation, with subsequent chapters relying strongly on results derived in the previous ones, any readers who 'dive in' part way through will have difficulty gaining a clear understanding of Barnsley's conclusions.

Despite this, the narrative unfolds in an almost jovial manner, allowing the author's contagious passion for the subject to shine through. Starting from mathematical first principles, Barnsley leads us through the evo-

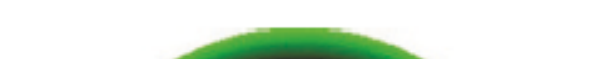

lution of algebraic sophistication and on to new and wondrous applications of the 'chaos game' and iterative system functions. Also known as a Markov chain Monte Carlo algorithm, this scheme provides a connection to basic fractals, such as the Sierpinski triangle. More importantly, the author shows how randomness might play a crucial role both in determining the rich complexity in nature and in the revolutionary field of bioinformatics.

The interplay between abstraction and the 'real world' is accented by Barnsley's set-theoretic formalism, but this makes it surprisingly easy to understand the digitization techniques, image transformations, and formation of selfsimilar fractal features. Computer-generated superfractals are interlaced with the equally artistic mathematical body of theorems, proofs and functional mechanisms that define the beauty within the self-similarity. These images encapsulate the transformations being described by the mathematics, exemplifying the fractal dynamics at work without any need for the technical jargon. In a sense, the reader is treated to two books at once, thanks to Barnsley's union of art and mathematics.

The occurrence of superfractal structure in the real world is highlighted by the author's choice of figures. The functions and graphs one might expect to find in a mathematicallyintensive treatise are nowhere to be seen. Instead, the pages are adorned with colourful pictures of leaves and animals, and there are even some photographs. This relaxed use of graphics is no accident; rather, it is a consequence of the underlying theme of the book, that the world is fundamentally described by the simple rules that dictate the chaotic overtures of iteration. It is clear from these images, as well as those on the companion website (www.superfractals.com), that Barnsley and colleagues have stumbled across a graphical technique that reaches

far into the burgeoning field of fractal aesthetics. The applications of superfractals in the computer graphics industry are obvious, and stand to transform the way in which textures and natural patterns are mimicked.

Overall, Superfractals would be a superb addition to the bookshelves of any scientists who use fractal analysis techniques in their research, be they physicist, biologist or economist. The author concludes by promising that the introduction of superfractals will revolutionize the way mathematics, physics, biology and art are combined, to produce a unified description of the complex world in which we live. After reading this book, I have no doubt that he is correct.

J.R. Mureika is in the Department of Physics, Loyola Marymount University, Los Angeles, California 90045, USA. 